\title{
Electroacupuncture Upregulated Ghrelin in Rats with Functional Dyspepsia via AMPK/TSC2/Rheb-Mediated mTOR Inhibition
}

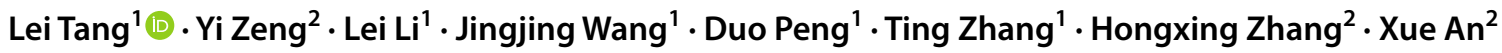

Received: 14 October 2018 / Accepted: 13 November 2019 / Published online: 21 December 2019

(c) The Author(s) 2019

\begin{abstract}
Background Gastrointestinal motility disorder is an important pathological basis for functional dyspepsia (FD). Epigastric ache and discomfort are the main symptoms of FD, and ghrelin deficiency is closely related to the occurrence and development of FD. While electroacupuncture (EA) alleviated the symptoms of FD patients and improved their quality of life, there is a lack of sufficient mechanistic evidence to support these beneficial effects.

Methods An in vivo FD model was established in wild-type and mammalian target of rapamycin (mTOR) knockout (-/-) rats. FD rats were subjected to EA with or without mTOR agonists or inhibitors. Gastric emptying and intestinal propulsion were assessed, and pathological changes in the hypothalamus, gastric antrum, and small intestine were examined histologically. In addition, ghrelin expression and AMPK/TSC2/Rheb/mTOR activation were detected by quantitative reverse transcription polymerase chain reaction and western blot.

Results EA alone or in combination with mTOR inhibitors improved gastrointestinal function in FD rats by increasing the rates of intestinal propulsion and gastric emptying, and pathological changes in the hypothalamus, gastric antrum, and small intestine were alleviated. This may be related to the significant upregulation of ghrelin expression and the effective activation of the AMPK/TSC2/Rheb/mTOR signaling pathway. Interestingly, EA also improved gastrointestinal function and ghrelin expression in mTOR (-/-) KO FD rats.

Conclusion Altering the level of ghrelin by regulating AMPK/TSC2/Rheb-mediated mTOR inhibition is an important way through which EA treats FD. The complex EA-mediated regulatory mechanisms of the brain-gut axis still require further exploration.
\end{abstract}

Keywords Electroacupuncture $\cdot$ Ghrelin $\cdot$ Functional dyspepsia $\cdot$ AMPK/TSC2/Rheb-mediated mTOR inhibition $\cdot$ Braingut axis

\section{Introduction}

Lei Tang and Yi Zeng have contributed equally to this paper.

Xue An

3350871798@qq.com

Hongxing Zhang

zhxzj99@aliyun.com

1 Rehabilitation Department, Central Hospital of Wuhan, Tongji Medical College, Huazhong University of Science and Technology, Wuhan, China

2 Department of Acumoxa, Wuhan Integrated TCM \& Western Medicine Hospital, Wuhan, China
As defined by the Rome III Diagnostic Criteria for Functional Gastrointestinal Disorders, functional dyspepsia (FD) is the presence of symptoms originating from the gastroduodenal region in the absence of any organic, systemic, or metabolic disease [1]. Epigastric aching and discomfort are the hallmark symptoms of FD. Most patients do not complain about pain, but rather suffer from burning, pressure, or fullness in the epigastric area to the extent where they cannot finish a normal meal [2]. Other common symptoms of FD include repeated and persistent postprandial nausea, belching, and abdominal bloating, which affect the quality of life [3]. According to epidemiological data, the incidence of FD in the worldwide population is $10-30 \%$ [4], with the highest prevalence in Asian countries such as China and India 
[5]. Though multiple pathogenic mechanisms have been proposed for $\mathrm{FD}$, its underlying etiology remains unclear.

Gastrointestinal motility disorder is an important pathological basis for FD [6]. Ghrelin, an important gastrointestinal hormone, is the only presently known endogenous ligand of growth hormone secretagogue receptor [7]. Ghrelin is also known as motilin-related peptide because it shares many functions with motilin including appetite control, stimulation of growth hormone release, gastric emptying and motility, and suppression of inflammation [8]. In addition to the main gastrointestinal tissues, ghrelin is reportedly detectable in the hypothalamus, pituitary gland, placenta, salivary gland, and other tissues [9]. Ghrelin deficiency in gastrointestinal tissues has been closely linked to the occurrence and development of FD in clinical examination [10], and abnormalities in plasma ghrelin levels may affect gut motility in FD patients [11]. Evidence has suggested that acyl ghrelin may potentially be used as an exogenous prokinetic agent for the treatment of slow transit in the upper gastrointestinal tract [12]. At present, several drugs that regulate gastrointestinal motility, visceral sensory properties, anxiety, and depression are used in the clinical treatment of FD [13]. Because of the complex and diverse pathogenesis of FD, its clinical manifestations vary greatly, and it is difficult for drug treatment to adapt to the complex individual differences. Thus, novel alternative therapies for FD are urgently needed.

Acupuncture is a form of therapy that has been applied in China for thousands of years to treat various psychiatric conditions [14]. It is frequently used to manage FD and has received favorable feedback from clinical practitioners because of its unique advantages. Acupuncture has alleviated the clinical symptoms of FD patients and improved their quality of life by modulating the homeostatic afferent network [15]. Electroacupuncture (EA) is a modification of acupuncture whereby electrodes are attached to the inserted needles to deliver a pulsed electric current. The scientific development of EA has made it clinically applicable against pain [16], depression [17], drug addiction [18], cerebrovascular trauma [19], anxiety [20], and gastrointestinal disorders [21]. Remarkably, stimulation of the "Zusanli" acupoint (tibialis anticus motor point) by acupuncture has long been advocated for the treatment of both diarrhea and constipation, with significant effects reported in both animals and humans [22, 23]. However, researchers have commented on the lack of sufficient evidence to support the beneficial effects of EA on FD because the metaphysical concepts of EA, which are based on theories of traditional Chinese medicine such as "qi" (air flow) and blood stasis, have gained limited acceptance. Therefore, clarifying the possible effects of EA on FD and the underlying mechanisms involved therein using well-characterized models may be of clinical benefit.

The mammalian target of rapamycin (mTOR) signaling pathway is involved in the regulation of a variety of biological phenomena and conditions, including aging and cancer. In terms of gastrointestinal function, mTOR is reportedly associated with gastric cancer progression [24], and gastric mTOR expression was shown to be linked to ghrelin production and energy intake [25]. We previously showed that low expression of ghrelin and highly phosphorylated mTOR existed in the gastrointestinal tissues of experimental rats subjected to FD [26]. However, it is unclear whether EA can affect gastrointestinal motility disorder caused by the abnormal expression of mTOR. In the current study, we established an in vivo model of FD using wild-type (WT) and mTOR (-/-) knockout (KO) rats and assessed the effects of EA on gastrointestinal function. We discuss the impact of EA on the regulation of the brain-gut axis and its mechanism, aiming to establish new therapies and a theoretical basis for clinical FD treatment.

\section{Materials and Methods}

\section{Animals}

Adult male WT Sprague-Dawley rats were provided by the experimental animal center of Hubei Institute of Medical Science. mTOR (-/-) KO rats were purchased from Model Animal Research Center of Nanjing University. All rats were maintained under controlled temperature $\left(22 \pm 1{ }^{\circ} \mathrm{C}\right)$ and humidity $(60 \% \pm 15 \%)$ in a 12-h light/dark cycle, given free access to deionized water, and fed irradiated disinfectant food. The rats were allowed to adapt to the above-mentioned conditions for seven days before experimentation.

\section{Construction and Assessment of FD Model}

All animal experiments were performed in accordance with the guidelines of the National Institutes of Health and were approved by the institutional review board. The FD model was established in WT and mTOR (-/-) KO rats via the tail clamping approach as previously described [27]. This model was first proposed by Guo et al. [28] and was intended to mimic the characteristics and elicit the symptoms of FD by inducing stress, as behaviors of stress (mood swings, depression, and anxiety) have been identified as strong factors in FD. Six rats were kept in one cage, and the distal third of the tail was clamped using surgical forceps while ensuring that the skin was not damaged. This resulted in responses of stress and anger among the rats due to pain and discomfort, in turn provoking them to fight violently with each other within the cage. This tail clamping procedure was practiced every $3 \mathrm{~h}$ for a duration of $30 \mathrm{~min}$, three times a day for seven days. Indications of successful modeling included hair loss, loose stools, reduction in diet, decreased activity, and drowsiness. 


\section{Animal Grouping and Treatment}

Thirty WT rats were randomized into five groups: normal, FD, FD+EA, FD+EA plus mTOR agonist (L-leucine), and FD+EA plus mTOR inhibitor (rapamycin). Twelve mTOR $(-/-) \mathrm{KO}$ rats were randomly divided into two groups: mTOR (-/-) KO+FD and mTOR (-/-) KO+FD + EA. For EA treatment, the rats were immobilized without anesthesia. The "Zusanli" acupoint was identified and routinely disinfected according to experimental acupuncture. EA was performed at the designated acupoint using an acupuncture needle $(0.28 \mathrm{~mm} \times 25 \mathrm{~mm})$ attached to a Korean acupoint nerve stimulator (frequency $4 \mathrm{~Hz}$; intensity $2 \mathrm{~mA}$; depth $7 \mathrm{~mm}$ ). The absence of obvious discomfort or rejection of rat limbs was considered to be the most appropriate condition. Each session lasted for $20 \mathrm{~min}$ and was performed once a day, six times a week for 4 weeks. For additional drug treatment, each rat was subjected to intraperitoneal administration of an mTOR agonist (L-leucine $0.45 \mathrm{~g} / \mathrm{kg} / \mathrm{d}[26,29]$ ) or inhibitor (rapamycin $1 \mathrm{mg} / \mathrm{kg} / \mathrm{d}[26,30]$ ) for four weeks. Normal (WT and mTOR (-/-) KO) rats did not receive any treatment.

\section{Measurement of Intestinal Propulsion and Gastric Emptying}

After four weeks of treatment, the changes in intestinal propulsion and gastric emptying were evaluated as described previously [31]. Three rats in each group were randomly selected and given 5\% graphite powder with milk and glucose in water (weighed and recorded as W1) by gavaging. The liquid was absorbed for $30 \mathrm{~min}$, after which the rats were killed. After exposure by laparotomy, the stomach and small intestine were carefully removed to observe the leading edge of the graphite powder in the intestine after ligation of the esophagogastric, gastroduodenal, and ileocecal junctions. The length of the small intestine from the pylorus to the ileocecal junction $(L 1)$ and the length of graphite powder $(L 2)$ were measured. Intestinal propulsion was calculated using the following formula: intestinal propulsion rate $=L 2 / L 1 \times 100 \%$. The stomach was cut, weighed (W2), and immersed in $0.9 \%$ saline solution to clean the rest of the graphite powder. After dry-blotting with absorbent paper to remove any surface moisture, the stomach was weighed again (W3). Gastric emptying rate was calculated using the following formula: gastric emptying rate $=(W 2-W 3) / W 1 \times 100 \%$.

\section{Tissue Preparation}

After four weeks of treatment, the remaining rats were euthanized by intraperitoneal injection of $100 \mathrm{mg} / \mathrm{kg}$ pentobarbital. The hypothalamus, gastric antrum, and small intestine were removed and fixed in 10\% buffered formalin until histological observation or frozen rapidly in liquid nitrogen and stored at $-80^{\circ} \mathrm{C}$ until further use.

\section{Hematoxylin and Eosin Staining}

Portions of the hypothalamus, gastric antrum, and small intestine were fixed in $10 \%$ buffered formalin for at least $24 \mathrm{~h}$, dehydrated with a sequence of ethanol solutions, and processed for paraffin embedding. Sections of $5 \mu \mathrm{m}$ in thickness were cut, deparaffinized, rehydrated, stained with hematoxylin and eosin, and examined under a light microscope (Olympus, Tokyo, Japan).

\section{Quantitative Reverse Transcription Polymerase Chain Reaction (qRT-PCR)}

Tissues from the hypothalamus, gastric antrum, and small intestine were ground in liquid nitrogen. Total RNA was extracted from frozen intestinal tissues using Trizol (15596018, Invitrogen, Carlsbad, CA, USA) and reversetranscribed into cDNA using the PrimeScript RT Master Mix (RR036Q, Takara Biotechnology, Dalian, China) according to the manufacturer's instructions. qRT-PCR was performed in a $20 \mu \mathrm{L}$ reaction mixture containing SYBR Green PCR Master Mix (4309155, Applied Biosystems), cDNA, and each primer at $0.2 \mathrm{mmol} / \mathrm{L}$ at $95^{\circ} \mathrm{C}$ for $10 \mathrm{~min}$, 40 cycles at $95{ }^{\circ} \mathrm{C}$ for $10 \mathrm{~s}$, and $60{ }^{\circ} \mathrm{C}$ for $45 \mathrm{~s}$. The data were collected using the QuantStudio ${ }^{\text {TM }} 6$ Flex Real-Time PCR System (Applied Biosystems). The relative amount of each gene was normalized to the housekeeping gene GAPDH and analyzed using the $2^{-\Delta \Delta C t}$ method. The primer sequences were: ghrelin F, 5'-CCAGCAGAGAAAGGA-3', R, 5'-CAACATCGAAGGGAG-3'; adenosine monophosphate-activated protein kinase (AMPK) F, 5'-ATGAGGTGG TGGAGCAG-3', R, 5'-AAGCGGAGGACAAAGTG-3'; tuberous sclerosis complex 2 (TSC2) F, 5'-AGTGGAGGC ACTTTGGA-3', R, 5'-ATGGAGGTCTTCGTTGG-3'; Ras homolog enriched in brain (Rheb) F, 5'-CCTCAGACATAC TCCAT-3', R, 5'-ATTTCCGACTAACATAA-3'; GAPDH F, 5'-CAGCAACAGGGTGGTGGA-3', R, 5'-TTGAGGGTG CAGCGAACT-3'.

\section{Protein Extraction and Western Blot}

Total proteins from the hypothalamus, gastric antrum, and small intestine tissues were extracted using a total protein extraction kit (Jiancheng Bioengineering Institute, Nanjing, China) and quantified by the bicinchoninic acid assay. Whole proteins were electrophoresed in $10-15 \%$ polyacrylamide gels. The separated proteins were transferred to polyvinylidene difluoride membranes, and non-specific binding was blocked with $5 \%$ skimmed milk for $2 \mathrm{~h}$ at $37^{\circ} \mathrm{C}$. The 
membranes were then incubated at $4{ }^{\circ} \mathrm{C}$ overnight with goat anti-ghrelin (Sc10368, 1:500, Santa Cruz Biotechnology Inc.), rabbit anti-p-AMPK (Sc33524, 1:1000, Santa Cruz Biotechnology Inc.), rabbit anti-p-TSC2 (ab52962, 1:10,000, Abcam), and rabbit anti-Rheb (ab25873, 1:1000, Abcam). GAPDH $(2118,1: 1000$, CST) was used as a control. Next, the membranes were incubated with goat anti-rabbit IgG (PAB150011, 1:10,000, Bioswamp) or donkey anti-goat IgG (PAB 150012, 1:10,000, Bioswamp) for $1 \mathrm{~h}$ at room temperature. Images were acquired using a multifunctional gel imaging system (Image Quant LAS 500, General Electric, Fairfield, CT, USA) after incubation with enhanced chemiluminescence reagent (P0018A, Shanghai Beyotime Biotechnology Co., Ltd., China).

\section{Statistical Analyses}

All values are presented as the mean \pm standard deviation. One-way analysis of variance followed by the Tukey's post hoc test was performed to compare differences between multiple groups (more than two) using SPSS 19.0 software (IBM Corp., Armonk, NY, USA). $P<0.05$ is considered statistically significant.

\section{Results}

\section{Rates of Intestinal Propulsion and Gastric Emptying}

In rats subjected to $\mathrm{FD}$, the rate of intestinal propulsion (Fig. 1a) was decreased $(P<0.01)$ and gastric emptying (Fig. 1b) slowed down significantly $(P<0.01)$ compared

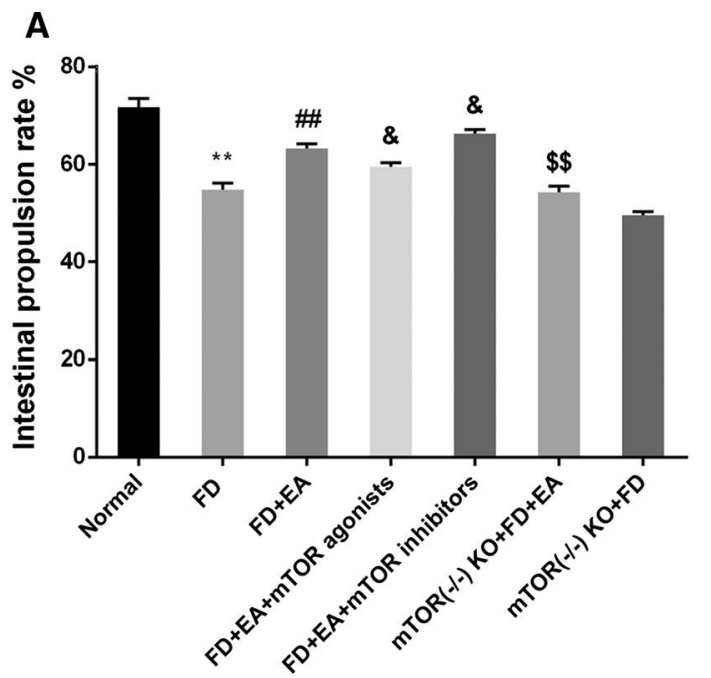

Fig. 1 EA increased the rates of intestinal propulsion and gastric emptying in FD rats. Changes in $\mathbf{a}$ intestinal propulsion rate and $\mathbf{b}$ gastric emptying rate. $F D$ functional dyspepsia, $E A$ electroacupunc- with those in normal rats. In turn, EA treatment reversed the effects of FD by increasing the rates of intestinal propulsion and gastric emptying $(P<0.01)$. With EA treatment, the administration of mTOR agonists decreased the rates of intestinal propulsion and gastric emptying $(P<0.05)$, whereas mTOR inhibitors increased them $(P<0.05)$. In mTOR (-/-) KO rats subjected to FD, EA also improved intestinal propulsion and gastric emptying $(P<0.01)$.

\section{Pathological Changes in the Hypothalamus, Gastric Antrum, and Small Intestine}

Histopathological observations of the hypothalamus, gastric antrum, and small intestine are presented in Fig. 2. In terms of the hypothalamus, normal cells showed uniform size, regular shape, and clear boundaries, whereas FD resulted in a decrease in the number of neurons, varied cell size and shape, and deeply stained nuclei with vacuolar changes. EA treatment significantly alleviated the above pathological changes in rats subjected to FD. On the basis of EA treatment, administration of mTOR agonists and inhibitors resulted in the aggravation and alleviation of hypothalamic lesions, respectively. In addition, EA treatment did not alleviate the pathological changes in the hypothalamus of mTOR (-/-) KO rats subjected to FD.

In terms of the gastric antrum and small intestine, no obvious lesion was found in normal rats. In rats subjected to FD, the gastric mucosa became thin and fractured, and the substratum was hyperemic. Edema of epithelial cells was observed in the bowel with focal necrosis, exfoliation, and infiltration of a large number of inflammatory cells. The gastrointestinal mucosa of rats treated by EA (with or without drugs) was more

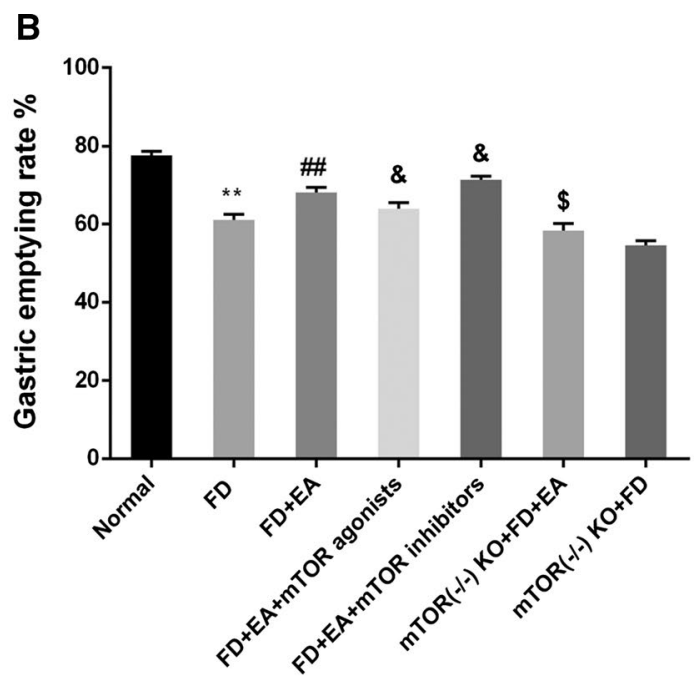

ture, $K O$ knockout. Compared with normal, $* * P<0.01$; compared with FD, ${ }^{\# \#} P<0.01$; compared with $\mathrm{FD}+\mathrm{EA},{ }^{\&} P<0.05$; compared with mTOR (-/-) KO+FD, ${ }^{\$} P<0.05,{ }^{\$ \$} P<0.01$ 
or less normal, with only slight hyperemia and inflammatory cell infiltration. Likewise, the epithelial cell structure was more or less normal. Nevertheless, the effect of EA on alleviating gastrointestinal pathological changes was not obvious in the mTOR (-/-) KO rats.

\section{mRNA Levels of Key Targets in the AMPK/TSC2/Rheb Signaling Pathway}

The mRNA levels of ghrelin, AMPK, TSC2, and Rheb in the hypothalamus, gastric antrum, and small intestine are indicated in Fig. 3. Rats subjected to FD showed decreased levels of ghrelin, AMPK, and TSC2 mRNA levels in the three types of tissues, whereas that of Rheb was increased (all $P<0.01$ ). In turn, single EA treatment increased the mRNA levels of ghrelin, AMPK, and TSC2 and decreased that of Rheb (all $P<0.01)$. Interestingly, both mTOR agonists and inhibitors reduced the mRNA levels of ghrelin, AMPK, and TSC2 to varying degrees after single EA treatment $(P<0.05, P<0.01)$. In addition, mTOR agonists significantly increased the mRNA level of Rheb compared with single EA treatment, whereas mTOR inhibitors did the opposite (both $P<0.01$ ). Surprisingly, EA treatment increased the mRNA levels of ghrelin, AMPK, and TSC2 in mTOR (-/-) KO rats subjected to FD $(P<0.01)$, but had no effect on Rheb.

\section{Protein Levels of Key Targets in the AMPK/TSC2/ Rheb Signaling Pathway}

The protein levels of ghrelin, p-AMPK, p-TSC2, and Rheb in the hypothalamus, gastric antrum, and small intestine are indicated in Fig. 4, showing certain similarities to mRNA levels. Typical AMPK/TSC2/Rheb signaling pathway inactivation and low-level ghrelin were observed in the three types of tissues in rats subjected to FD compared to those in normal rats (all $P<0.01$ ). Correspondingly, EA treatment significantly increased the protein level of ghrelin, p-AMPK, and p-TSC2 while diminishing the expression of Rheb (all $P<0.01$ ). Compared with single EA treatment, mTOR inhibitors further promoted the effect of EA to a certain extent, whereas mTOR agonists did the opposite (all $P<0.01$ ). However, this effect did not apply to Rheb, as both mTOR agonists and inhibitors increased its protein level (all $P<0.01$ ). Surprisingly, in mTOR (-/-) KO rats subjected to FD, EA treatment increased the protein expression of ghrelin and p-AMPK $(P<0.01)$, but had no effect on p-TSC2 and Rheb.

\section{Discussion}

Our study demonstrated the regulation of AMPK/ TSC2/Rheb-mediated mTOR inhibition by EA in altering the expression of ghrelin, which acted on the hypothalamic-intestinal axis to improve the gastrointestinal function of rats subjected to FD. This conclusion is supported by the following observations: (1) FD resulted in low expression of ghrelin and poor gastrointestinal function; (2) EA significantly upregulated ghrelin levels, increased the rates of intestinal propulsion and gastric emptying, and attenuated damage to the hypothalamus, gastric antrum, and small intestine in FD rats; (3) mTOR inhibitors amplified the therapeutic effects of EA against FD, whereas mTOR agonists did the opposite; (4) EA activated AMPK/TSC2/Rheb-mediated mTOR inhibition in FD rats, which has synergistic effects with mTOR inhibitors; and (5) EA significantly increased ghrelin expression and the level of phosphorylated AMPK in mTOR (-/-) KO FD rats and further improved gastrointestinal function, but had no effect on the expression of downstream targets of the AMPK signaling pathway.

FD impacts patients' quality of life and imparts significant economic burden to the healthcare system because of ineffective treatment [32]. Current therapeutic options and management of FD focus on diet regulation, psychological therapies, anti-nociceptive agents, antidepressants, prokinetic agents, and complementary and alternative medicine [13]. As a form of alternative therapy with a long history, traditional Chinese acupuncture is based on the metaphysical concept of "qi," a supposed body energy that runs through hypothesized channels called meridians. There are 365 designated acupuncture points on these meridians that can be used for stimulation via needles to balance "yin" and "yang" by relieving blockages in the flow of "qi" [14]. Among them, the "Zusanli" in the current study is a commonly used and safe acupoint that has been repeatedly verified by multiple clinical treatments. For instance, EA on the "Zusanli" acupoint suppressed inflammation in allergic contact dermatitis by triggering local interleukin-10 production and inhibiting p38 mitogen-activated protein kinase activation [33]. In terms of combination therapy, bone marrow-derived mesenchymal stem cells combined with the EA stimulation of "Zusanli" protected the intestinal mucosal barrier from intestinal ischemia/ reperfusion [34]. In our study, the optimal needle depth and the intensity of the electric current were defined on the basis of ethics and morality in preliminary experiments. We found that the gastrointestinal function of FD rats was significantly improved with EA treatment, which was mainly manifested in the increased rates of intestinal propulsion and gastric emptying and the alleviation of hypothalamic-gastrointestinal injury. We concluded that this may be partially or indirectly related to the increase in ghrelin expression in the hypothalamus, gastric antrum, and small intestine, in turn resulting in the regulation of the intricate brain-intestinal axis. This is an important aspect that contributes to the pathogenesis of FD, and in 


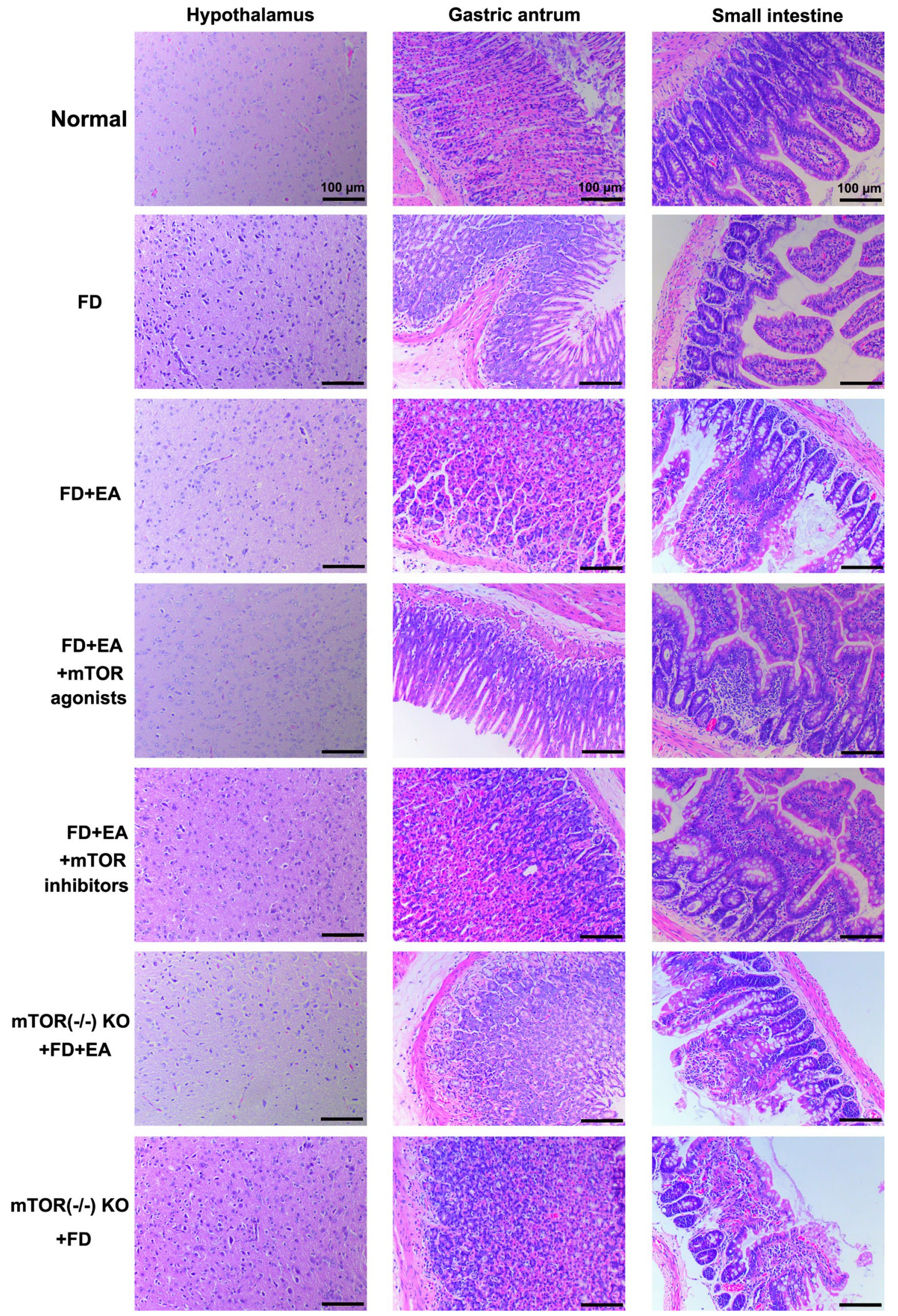


4Fig. 2 EA alleviated the pathological injury in the hypothalamus, gastric antrum, and small intestine tissues in FD rats. $F D$ functional dyspepsia, $E A$ electroacupuncture, $K O$ knockout. Scale bar: $100 \mu \mathrm{m}$

stimulating the "Zusanli" acupoint, EA effectively modulated the brain-gut axis, thus exerting an effect on FD. In patients with gastroparesis or gastric dysrhythmia, the stimulation of the "Zusanli" acupoint by EA increased the regularity of slow waves in the stomach, the speed of liquid gastric emptying, and the activity of the vagal nerve [35]. These results indicate that the stimulation of the "Zusanli" acupoint by EA is a safe and effective method to treat FD.

During EA treatment, we found that the increase in ghrelin level was accompanied by the activation of the AMPK/ TSC2/Rheb signaling pathway. In addition, mTOR inhibitors promoted the effect of EA therapy. We speculated that this effect may be achieved by regulating AMPK/TSC2/Rhebmediated mTOR inhibition, so we applied an mTOR agonist (L-leucine) or inhibitor (rapamycin) to assist EA in the treatment of FD rats. Similar to what we expected, mTOR inhibitors further increased the rates of intestinal propulsion and gastric emptying in EA-treated FD rats, whereas mTOR agonists did the opposite. Interestingly, this process was still accompanied by changes in ghrelin level and AMPK/ TSC2/Rheb signaling. We concluded that feedback regulation exists between mTOR-mediated ghrelin and AMPK/ TSC2/Rheb signaling. mTOR is a central regulator of cell growth, aging, ribosome biogenesis, protein synthesis, autophagy, and metabolism [36]. mTOR signaling in hypothalamic neurons has been shown to be involved in neuronal sensing of nutrient availability, regulated food intake, and energy balance [37]. Ghrelin, the only currently identified circulating hormone, is able to induce the sensation of hunger and initiate food intake via the central nervous system, which involves the hypothalamus [38]. Multiple studies have shown that mTOR signaling mediates the orexigenic action of ghrelin. For example, in a study of mouse obesity, inhibition of gastric mTOR signaling by rapamycin stimulated the mRNA expression of ghrelin and increased plasma ghrelin, while activation of gastric mTOR signaling by L-leucine decreased the level of plasma ghrelin [25]. The current study used EA to link the AMPK/TSC2/Rheb-mediated mTOR inhibition to ghrelin activity and demonstrated that direct control of ghrelin expression occurred in the hypothalamus, gastric antrum, and small intestine, signifying that mTOR is a crucial control point in ghrelin expression. However, it is noteworthy that mTOR (-/-) KO FD rats treated by EA also showed upregulated ghrelin expression, which may be related to other EA-mediated signal regulation pathways.

Rheb, a small GTP-binding protein, relays upstream signals to regulate mTOR activity positively. It reportedly binds directly to the amino terminal lobe of the mTOR catalytic domain to activate mTOR kinase in a GTP/GDP-dependent manner [39]. TSC1/2 is a heterodimer complex that inhibits the activity of mTOR complex 1 by transforming Rheb-GTP into the GDP coupling form Rheb-GDP and is a classical inhibiting factor of mTOR as well as a downstream protein in the AMPK pathway [40]. AMPK is an important regulator of intracellular energy metabolism, which is widely discovered in various tissues such as skeletal muscle, gastrointestinal tissues, and pancreas. When AMPK is phosphorylated and activated, it shuts down the anabolic metabolic pathway that consumes adenosine triphosphate (ATP). On the other hand, it opens up the catabolic metabolic pathway that produces ATP, accurately reflecting the changes in cell energy state [41]. Activated AMPK can directly phosphorylate TSC-2, promote the formation of the TSC1/2 complex, and competitively inhibit the activity of mTORC1, showing a new regulatory pathway of AMPK/TSC2/Rheb-mediated mTOR inhibition. A large number of animal studies related to various diseases have shown that AMPK was an effective molecular target of EA. For example, EA attenuated hepatic lipid accumulation via AMPK activation in obese rats [42]. In mice with Alzheimer's disease, EA stimulation alleviated the memory impairment and increased the levels of p-AMPK in the hippocampus [43]. The phenomenon of EAregulated ghrelin level via AMPK activation also appeared in our study.

The disturbance of the brain-gut axis, which may be involved in the development of FD, has recently been revealed $[44,45]$. The brain-gut axis is a complex, bidirectional communication system between the gastrointestinal tract and the nervous system. It not only ensures gastrointestinal homeostasis, but is also closely related to motivation and cognitive functions, such as intuitive decision [46]. Emotional and psychological changes may affect the functions of the digestive system, which also apply to FD patients [47]. The normalization of the brain-gut axis is essential for FD treatment. In our study, seven days of continuous irritation promoted the successful establishment of the rat model of FD. Both the gastrointestinal tract and the neurons of the hypothalamus were pathologically damaged to different degrees. Importantly, the activation of AMPK signaling and the symptom improvement induced by EA stimulation of "Zusanli" are systemic, as reflected in the hypothalamus, gastric antrum, and small intestine. Using positron emission tomography-computed tomography, researchers identified that acupuncture affects brain activities and tends to normalize changes in brain activities in FD patients [48]. This is consistent with the amelioration of hypothalamic injury induced by EA in our study. In other research on FD rats, EA intervention by stimulating the "Zusanli" and "Taichong" acupoints promoted gastrointestinal motor ability, which has been linked to the downregulation of neurotensin in the plasma, hypothalamus, gastric antrum, and ileum [44]. The 
Fig. 3 EA increased the mRNA level of ghrelin and the expression of downstream targets of AMPK signaling pathway in the hypothalamus, gastric antrum, and small intestine tissues of FD rats. Changes in mRNA level of each target in the a hypothalamus, $\mathbf{b}$ gastric antrum, and $\mathbf{c}$ small intestine. $F D$ functional dyspepsia, $E A$ electroacupuncture, $K O$ knockout. Compared with normal, $* * P<0.01$; compared with FD, ${ }^{\# \#} P<0.01$; compared with $\mathrm{FD}+\mathrm{EA},{ }^{\&} P<0.05$,

${ }^{\& \&} P<0.01$; compared with mTOR (-l-) KO+FD,

${ }^{\$} P<0.01$

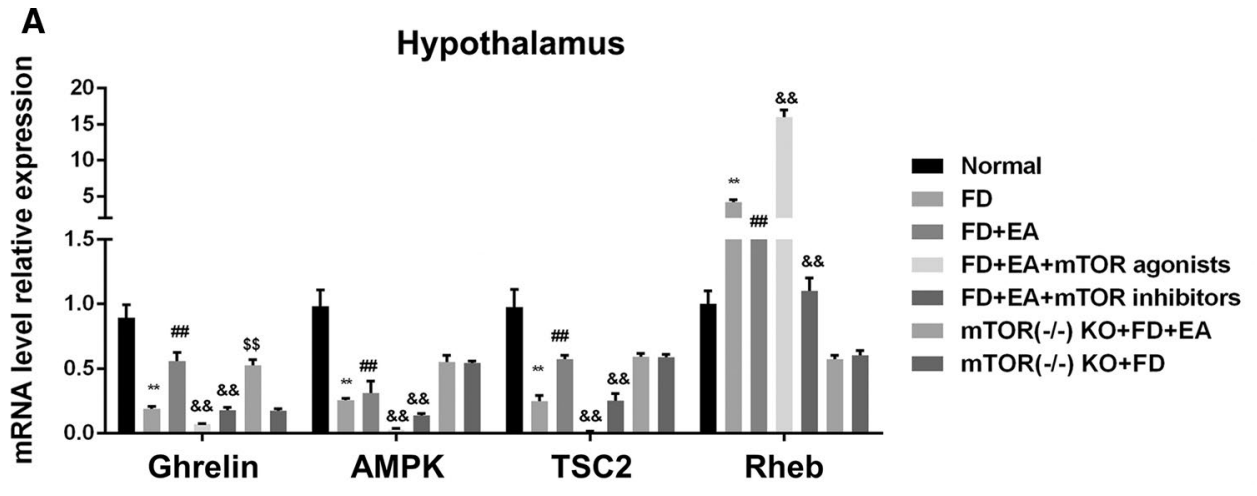

B

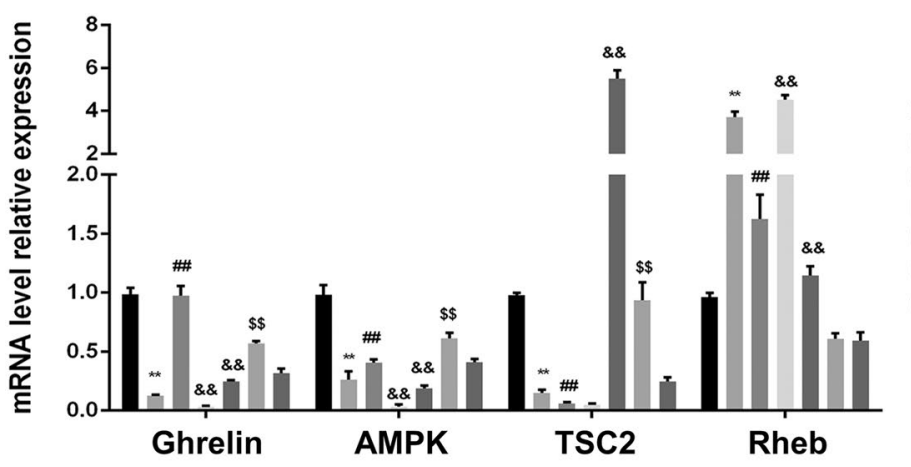

Normal
FD

- FD+EA

FD+EA+mTOR agonists

- FD+EA+mTOR inhibitors

mTOR(-/-) KO+FD+EA

mTOR(-/-) KO+FD

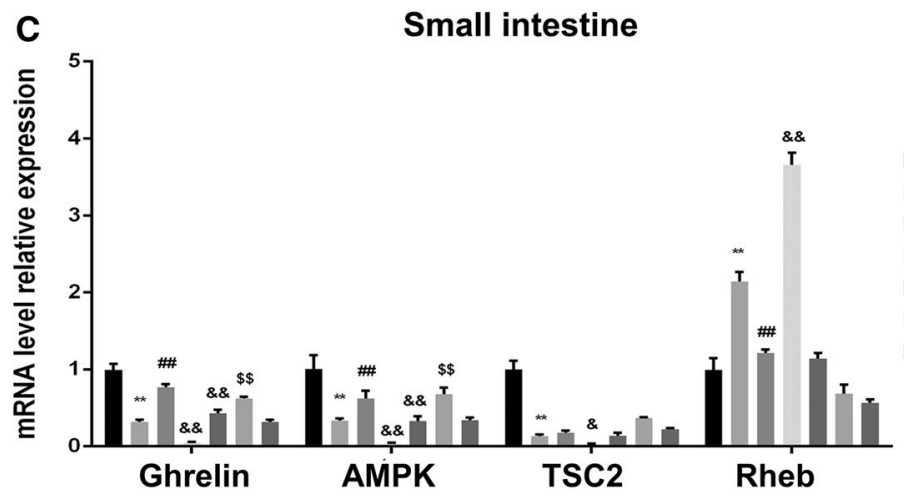

normal

- FD

- FD+EA

FD+EA+mTOR agonists

- FD+EA+mTOR inhibitors

mTOR(-/-) KO+FD+EA

mTOR(-/-) KO+FD

Ghrelin

AMPK

TSC2

Rheb

involvement of neurotensin in the brain-gut axis is suggested in EA-induced amelioration of FD.

In conclusion, our findings demonstrated that altering the level of ghrelin by regulating AMPK/TSC2/Rhebmediated mTOR inhibition is one of the mechanisms through which FD can be treated by EA. Future research will explore the impact of EA on the brain-gut axis in depth to lay a theoretical basis that eliminates the bias against the metaphysical aspects of EA. 

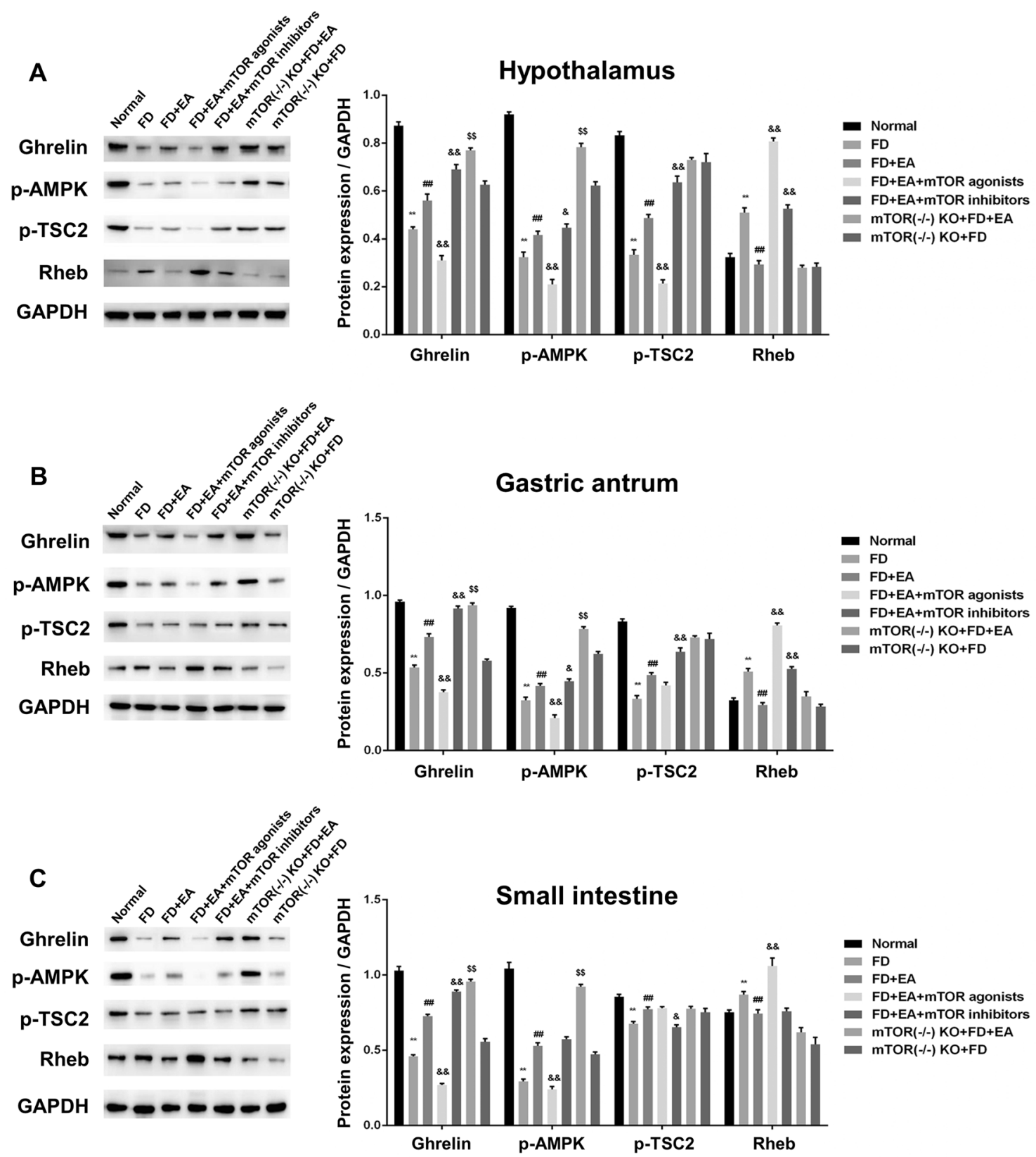

Fig. 4 EA increased the protein level of ghrelin and activated AMPK/ TSC2/Rheb-mediated mTOR inhibition in the hypothalamus, gastric antrum, and small intestine tissues of FD rats. Changes in protein level of each target in the a hypothalamus, b gastric antrum, and $\mathbf{c}$

Acknowledgments This study was funded by grants from the 2016 special foundation of Yellow Crane Meritocrat (Wuhan talent office [2017]2)

\section{Compliance with Ethical Standards}

Conflict and interest The authors declare that there are no conflicts of interest. small intestine. $F D$ functional dyspepsia, $E A$ electroacupuncture, $K O$ knockout. Compared with normal, $* * P<0.01$; compared with FD, ${ }^{\# \#} P<0.01$; compared with FD + EA treatment, ${ }^{\&} P<0.05,{ }^{\& \&} P<0.01$; compared with mTOR (-/-) KO+FD, ${ }^{\$ \$} P<0.01$

Open Access This article is distributed under the terms of the Creative Commons Attribution-NonCommercial 4.0 International License (http://creativecommons.org/licenses/by-nc/4.0/), which permits any noncommercial use, distribution, and reproduction in any medium, provided you give appropriate credit to the original author(s) and the source, provide a link to the Creative Commons license, and indicate if changes were made. 


\section{References}

1. Tack J, Talley NJ. Functional dyspepsia-symptoms, definitions and validity of the Rome III criteria. Nat Rev Gastroenterol Hepatol. 2013;10:134-141.

2. Karamanolis G, Caenepeel P, Arts J, Tack J. Association of the predominant symptom with clinical characteristics and pathophysiological mechanisms in functional dyspepsia. Gastroenterology. 2006;130:296-303.

3. Vanheel H, Carbone F, Valvekens L, et al. Pathophysiological abnormalities in functional dyspepsia subgroups according to the Rome III criteria. Am J Gastroenterol. 2017;112:132-140.

4. Olafsdottir LB, Gudjonsson H, Jonsdottir HH, Thjodleifsson B. Natural history of functional dyspepsia: a 10-year populationbased study. Digestion. 2010;81:53-61.

5. Ghoshal UC, Singh R, Chang FY, et al. Epidemiology of uninvestigated and functional dyspepsia in Asia: facts and fiction. $J$ Neurogastroenterol Motil. 2011;17:235-244.

6. Kusano M, Hosaka H, Kawada A, et al. Gastrointestinal motility and functional gastrointestinal diseases. Curr Pharm Des. 2014;20:2775-2782.

7. Ogiso K, Asakawa A, Amitani H, Inui A. Ghrelin: a gut hormonal basis of motility regulation and functional dyspepsia. $J$ Gastroenterol Hepatol. 2011;26:67-72.

8. Chen CY, Tsai CY. Ghrelin and motilin in the gastrointestinal system. Curr Pharm Des. 2012;18:4755-4765.

9. Ghelardoni S, Carnicelli V, Frascarelli S, Ronca-Testoni S, Zucchi R. Ghrelin tissue distribution: comparison between gene and protein expression. J Endocrinol Invest. 2006;29:115-121.

10. Takamori K, Mizuta Y, Takeshima F, et al. Relation among plasma ghrelin level, gastric emptying, and psychologic condition in patients with functional dyspepsia. J Clin Gastroenterol. 2007;41:477-483

11. Shinomiya T, Fukunaga M, Akamizu T, et al. Plasma acylated ghrelin levels correlate with subjective symptoms of functional dyspepsia in female patients. Scand J Gastroenterol. 2005;40:648-653

12. Akamizu T, Iwakura H, Ariyasu H, et al. Repeated administration of ghrelin to patients with functional dyspepsia: its effects on food intake and appetite. Eur J Endocrinol. 2008; 158:491-498.

13. Lacy BE, Talley NJ, Locke GR 3rd, et al. Review article: current treatment options and management of functional dyspepsia. Aliment Pharmacol Ther. 2012;36:3-15.

14. Ulett GA, Han S, Han JS. Electroacupuncture: mechanisms and clinical application. Biol Psychiat. 1998;44:129-138.

15. Zeng F, Qin W, Ma T, et al. Influence of acupuncture treatment on cerebral activity in functional dyspepsia patients and its relationship with efficacy. Am J Gastroenterol. 2012;107:1236-1247.

16. Wang WS, Tu WZ, Cheng RD, et al. Electroacupuncture and A-317491 depress the transmission of pain on primary afferent mediated by the $\mathrm{P} 2 \mathrm{X} 3$ receptor in rats with chronic neuropathic pain states. J Neurosci Res. 2014;92:1703-1713.

17. Han C, Li XW, Luo HC. Comparative study of electro-acupuncture and maprotiline in treating depression. Chin J Integr Tradit West Med. 2002;22:512-514, 521.

18. Wen HL, Teo SW. Experience in the treatment of drug addiction by electro-acupuncture. Hong Kong Nurs J. 1975;19:33-35.

19. Wong AM, Su TY, Tang FT, Cheng PT, Liaw MY. Clinical trial of electrical acupuncture on hemiplegic stroke patients. Am J Phys Med Rehabil. 1999;78:117-122.

20. Chae Y, Yeom M, Han JH, et al. Effect of acupuncture on anxiety-like behavior during nicotine withdrawal and relevant mechanisms. Neurosci Lett. 2008;430:98-102.
21. Liu MY, Wang CW, Wu ZP, Li N. Electroacupuncture for the prevention of postoperative gastrointestinal dysfunction in patients undergoing vascular surgery under general anesthesia: study protocol for a prospective practical randomized controlled trial. $J$ Integr Med. 2014;12:512-519.

22. Qin $\mathrm{Z}, \mathrm{Li} \mathrm{B}, \mathrm{Wu}$ J, et al. Acupuncture for chronic diarrhea in adults: protocol for a systematic review. Medicine (Baltimore). 2017;96:e5952.

23. Zheng H, Li Y, Zhang W, et al. Electroacupuncture for patients with diarrhea-predominant irritable bowel syndrome or functional diarrhea: a randomized controlled trial. Medicine (Baltimore). 2016;95:e3884.

24. Sun D, Toan X, Zhang Y, et al. Mammalian target of rapamycin pathway inhibition enhances the effects of 5-aza-dC on suppressing cell proliferation in human gastric cancer cell lines. Sci China C Life Sci. 2008;51:640-647.

25. Xu G, Li Y, An W, et al. Gastric mammalian target of rapamycin signaling regulates ghrelin production and food intake. Endocrinology. 2009;150:3637-3644.

26. Zeng $\mathrm{Y}$, Zhang H-X. The roles of mTOR agonist and mTOR inhibitor in electro-acupuncture treatment on functional dyspepsia. Liaoning J. Tradit. Chin. Med. 2018;2:405-409.

27. Chang X, Zhao L, Wang J, Lu X, Zhang S. Sini-san improves duodenal tight junction integrity in a rat model of functional dyspepsia. BMC Complement Altern Med. 2017;17:432.

28. Guo H, Lin J, Li G, Li Y, Tao X, Luo S. The animal model study of functional dyspepsia. Chin J Integr Tradit West Med Dig. 2001;9:141-142.

29. Yoshizawa F, Mochizuki S, Sugahara K. Differential dose response of mTOR signaling to oral administration of leucine in skeletal muscle and liver of rats. Biosci Biotechnol Biochem. 2013;77:839-842.

30. Lu Z, Liu F, Chen L, et al. Effect of chronic administration of low dose rapamycin on development and immunity in young rats. PLoS ONE. 2015;10:e0135256.

31. Liang Q, Yan Y, Mao L, et al. Evaluation of a modified rat model for functional dyspepsia. Saudi J Gastroenterol. 2018;24:228-235.

32. Hantoro IF, Syam AF, Mudjaddid E, Setiati S, Abdullah M. Factors associated with health-related quality of life in patients with functional dyspepsia. Health Qual Life Outcomes. 2018;16:83.

33. Wang Z, Yi T, Long M, et al. Electro-acupuncture at Zusanli acupoint (ST36) suppresses inflammation in allergic contact dermatitis via triggering local IL-10 production and inhibiting p38 MAPK activation. Inflammation. 2017;40:1351-1364.

34. Geng Y, Chen D, Zhou J, et al. Synergistic effects of electroacupuncture and mesenchymal stem cells on intestinal ischemia/ reperfusion injury in rats. Inflammation. 2016;39:1414-1420.

35. Chang CS, Ko CW, Wu CY, Chen GH. Effect of electrical stimulation on acupuncture points in diabetic patients with gastric dysrhythmia: a pilot study. Digestion. 2001;64:184-190.

36. Asnaghi L, Bruno P, Priulla M, Nicolin A. mTOR: a protein kinase switching between life and death. Pharmacol Res. 2004:50:545-549.

37. Cota D, Proulx K, Smith KA, et al. Hypothalamic mTOR signaling regulates food intake. Science. 2006;312:927-930.

38. Li Q, Yu Q, Lin L, et al. Hypothalamic peroxisome proliferatoractivated receptor gamma regulates ghrelin production and food intake. Neuropeptides. 2018;69:39-45.

39. Long X, Lin Y, Ortiz-Vega S, Yonezawa K, Avruch J. Rheb binds and regulates the mTOR kinase. Curr Biol. 2005;15:702-713.

40. Xie J, Ponuwei GA, Moore CE, Willars GB, Tee AR, Herbert TP. cAMP inhibits mammalian target of rapamycin complex-1 and -2 (mTORC1 and 2) by promoting complex dissociation and inhibiting mTOR kinase activity. Cell Signal. 2011;23:1927-1935. 
41. Viollet B, Guigas B, Leclerc J, et al. AMP-activated protein kinase in the regulation of hepatic energy metabolism: from physiology to therapeutic perspectives. Acta Physiol. 2009;196:81-98.

42. Gong M, Cao C, Chen F, et al. Electroacupuncture attenuates hepatic lipid accumulation via AMP-activated protein kinase (AMPK) activation in obese rats. Acupunct Med. 2016;34:209-214.

43. Dong W, Guo W, Zheng X, et al. Electroacupuncture improves cognitive deficits associated with AMPK activation in SAMP8 mice. Metab Brain Dis. 2015;30:777-784.

44. Yang Y, Xu PD, Xin Y, Kang ZX, Zhang HX, Zhou L. Involvement of neurotensin-mediated brain-gut axis in electroacupuncture intervention induced improvement of functional dyspepsia in rats. Acupunct Res. 2016;41:35-39, 50.

45. Tominaga $\mathrm{K}$, Tsumoto $\mathrm{C}$, Ataka $\mathrm{S}$, et al. Regional brain disorders of serotonin neurotransmission are associated with functional dyspepsia. Life Sci. 2015;137:150-157.
46. Kountouras J, Zavos C, Polyzos SA, Deretzi G. The gut-brain axis: interactions between Helicobacter pylori and enteric and central nervous systems. Ann Gastroenterol. 2015;28:506.

47. Dragos D, Ionescu O, Micut R, Ojog DG, Tanasescu MD. Psychoemotional features of a doubtful disorder: functional dyspepsia. J Med Life. 2012;5:260-276.

48. Fang J, Wang D, Zhao Q, et al. Brain-gut axis modulation of acupuncture in functional dyspepsia: a preliminary restingstate fcMRI study. Evid Based Complementary Altern Med. 2015;2015:860463.

Publisher's Note Springer Nature remains neutral with regard to jurisdictional claims in published maps and institutional affiliations. 